\title{
Infant Mortality Rates for Metropolitan and Nonmetropolitan Counties, * by Single Race and Hispanic Origin - National Vital Statistics System, United States, 2019
}

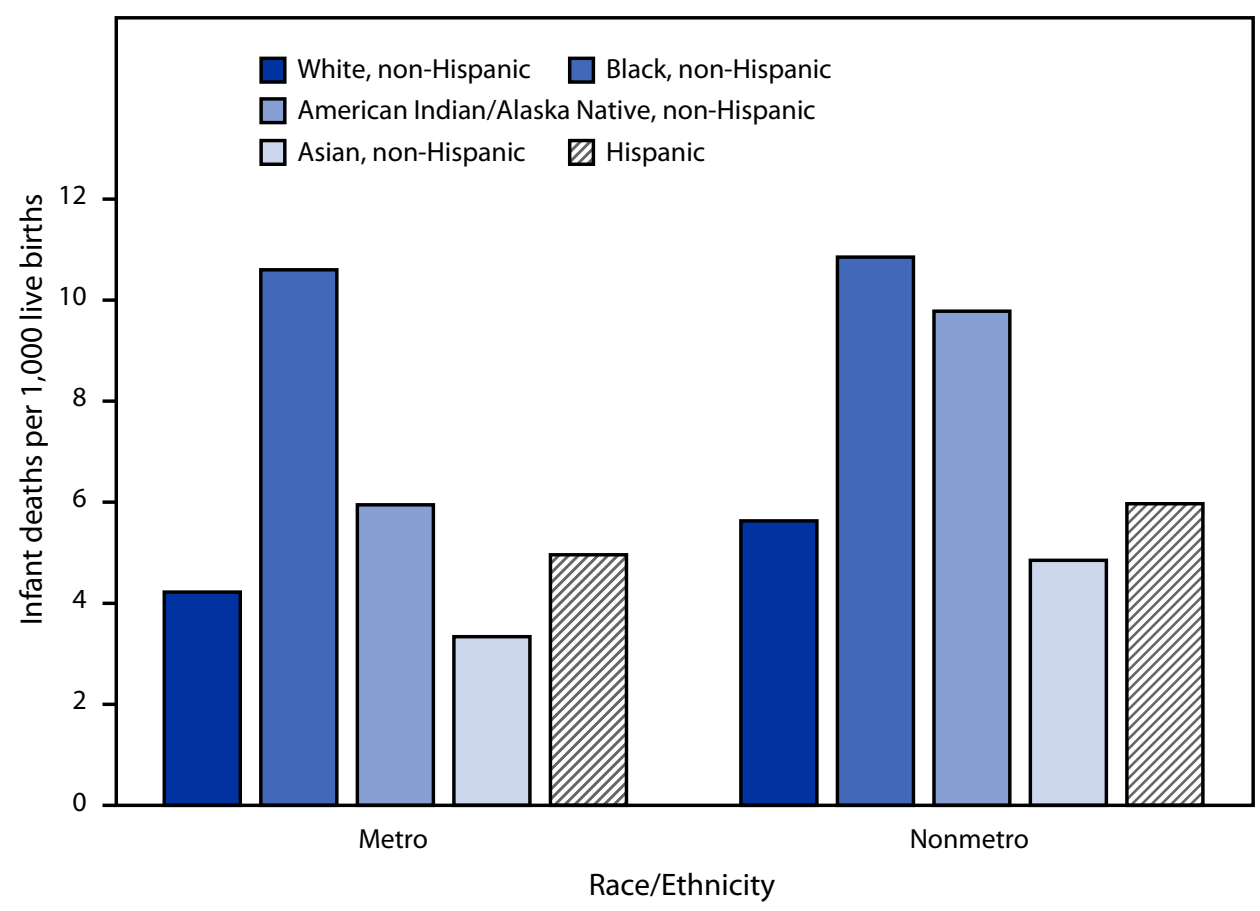

\footnotetext{
* Urbanization level is based on maternal county of residence. Counties were classified according to their metropolitan status using the National Center for Health Statistics Urban-Rural Classification Scheme. https://www.cdc.gov/nchs/data_access/urban_rural.htm
}

\begin{abstract}
In metropolitan counties, infant mortality rates were highest for infants of non-Hispanic Black mothers (10.60 infant deaths per 1,000 live births), followed by infants of non-Hispanic American Indian or Alaska Native (5.95), Hispanic (4.96), non-Hispanic White (4.22), and non-Hispanic Asian (3.34) mothers. In nonmetropolitan counties, the mortality rate was also highest for infants of non-Hispanic Black mothers (10.85), followed by infants of non-Hispanic American Indian or Alaska Native (9.78), Hispanic (5.97), non-Hispanic White (5.63), and non-Hispanic Asian (4.85) mothers. The infant mortality rate was significantly lower for infants of non-Hispanic White, non-Hispanic American Indian or Alaska Native, and Hispanic mothers in metropolitan counties compared with nonmetropolitan counties; differences in rates between metropolitan and nonmetropolitan counties for infants of non-Hispanic Black and non-Hispanic Asian mothers were not statistically significant.
\end{abstract}

Source: National Vital Statistics System. Linked Birth and Infant Death Data. https://www.cdc.gov/nchs/nvss/linked-birth.htm Reported by: Danielle M. Ely, PhD, dely@cdc.gov, 301-458-4812. 